\title{
A Security Specification Library with a Schemaless Database
}

\author{
Shoichi Morimoto ${ }^{1}$ and Jingde Cheng $^{2}$ \\ ${ }^{1}$ School of Industrial Technology, Advanced Institute of Industrial Technology \\ 1-10-40, Higashi-oi, Shinagawa-ku, Tokyo, 140-0011, Japan \\ morimoto-syoichi@aiit.ac.jp \\ 2 Department of Information and Computer Sciences, Saitama University \\ 255, Shimo-okubo, Sakura-ku, Saitama, 338-8570, Japan \\ cheng@aise.ics.saitama-u.ac.jp
}

\begin{abstract}
In order to develop highly secure information systems, it is important to make a security specification of the systems, although it requires heavy labor. Thus database technologies have been applied to software engineering and information security engineering for practical reuse of security specifications. However, because the specifications do not have fixed layout, it is difficult to develop a flexible and useful library for the documents with conventional database technologies. Therefore, this paper proposes a security specification library with a schemaless native XML database. Users of the library can directly store and manage security specifications with any layout. Consequently, the library mitigates the labor for making security specifications.
\end{abstract}

Keywords: Common criteria, Digital pattern processing.

\section{Introduction}

Because quality of security specifications in development process are directly and closely related to security of information systems, it is desirable, but difficult, to develop a high quality security specification. Thus, we have developed a security requirement management database with a relational database, which supports development of secure information systems [1,4. Users of the database can effectively manage and reuse security requirements in security specifications.

However, the specification documents severally have various styles and structures, because there are many software development processes and developers have to use the styles and structures suitable for each development process. Thus, it is difficult to develop a flexible and useful database for the purpose with relational or object-oriented databases that need to define fixed structure.

Therefore, in this paper we rebuild the security requirement management database with a schemaless native XML database. Users of the improved database can freely store security specifications without consideration to document structure after converting them into XML documents. Consequently, it mitigates labor making high quality security specifications and developing highly secure information systems. 


\section{The Security Requirement Management Database}

The security requirement management database, named ISEDS, can manage data of specifications which are designed in conformity to ISO/IEC 15408 common criteria 1, 4. The criteria define security functional requirements which should be applied to validate an information system [2. Applicants who apply to obtain the evaluation of ISO/IEC 15408 have to describe and submit a security specification of their system. The security specification, called security target, must describe the category of the target system which is evaluated (target of evaluation, TOE for short), threats which are anticipated in the TOE, security objectives to oppose the threats, security functional requirements for achievement of the objectives, and security functions for implementation of the requirements. ISEDS implements the components of security targets and their interrelationships with a relational database.

\section{Improvement of the Database}

We reimplemented ISEDS with NeoCore XML Management System (XMS) 7], which is a native XML database and free for development only. Its advantage is high performance and low resource utilization because of its patented Digital Pattern Processing (DPP) technology. With DPP, XMS describes XML documents and their component contents in numeric finite-width fields. This ensures that performance remains unaffected by the length of tag names, element content, or document hierarchy. To further improve performance, DPP enables XMS to "index" every tag, element, and combination, ensuring extremely fast searches

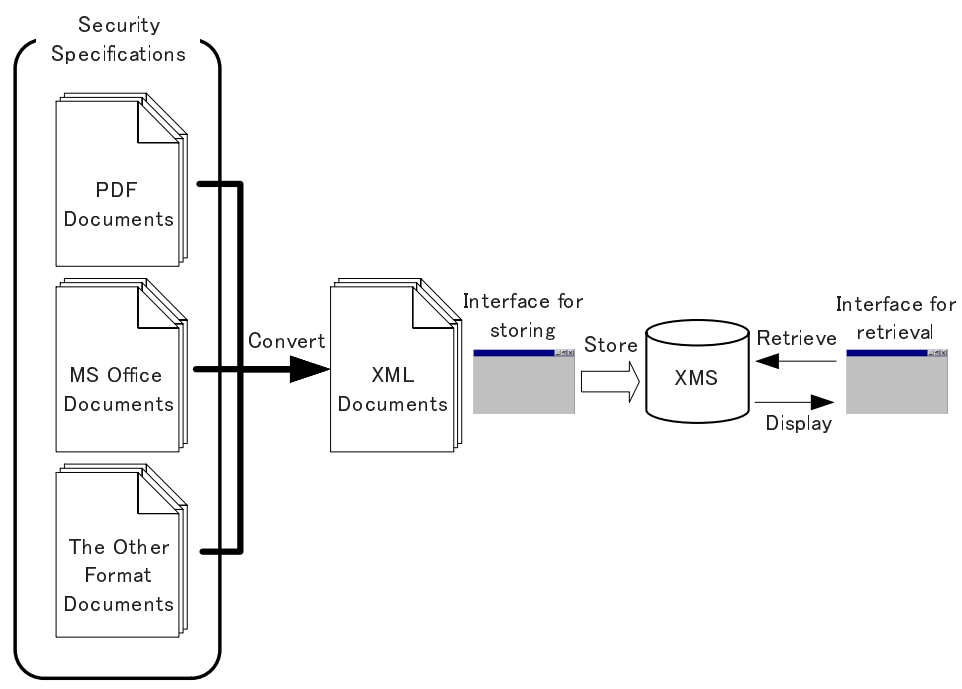

Fig. 1. The security requirement management database with XMS 


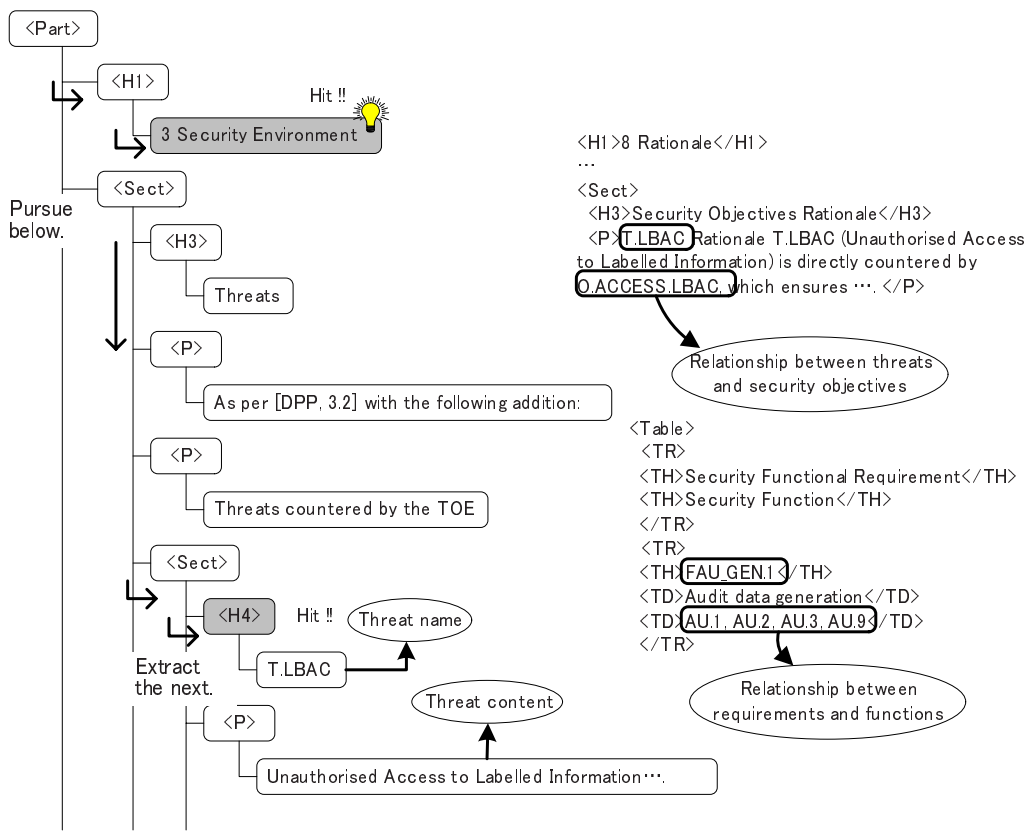

Fig. 2. The retrieval function behavior

on tags and data. The users of XMS can flexibly retrieve XML documents with XQuery, XPath, and FLWR expressions [6. The most important merit is that XMS does not require defining a schema. That is, XMS can directly store XML documents without a schema. Because these advantages can solve the problem of ISEDS, we adopted XMS.

The improved ISEDS can store any layout and format document. Users must first convert security specifications into XML documents. Specifications, perhaps, are generally written by MS Word, Excel, OpenOffice, or PDF. Adobe Acrobat can convert PDF files into XML documents. Office documents can do likewise. Somehow the other format documents are also convertible into XML. After the conversion, the users can store them into ISEDS via the user interface. The interface appends metadata to the XML documents and stores them into XMS. The metadata record timestamps, modification times, source file passes, identifications, and tag names of document captions for retrieval. One can easily retrieve, update and delete the stored data via the other interface (Fig. 1). Because each XML document tree in ISEDS is different, there are no fixed passes for retrieval. Therefore, ISEDS retrieval function distinguishes each component in security specifications by caption names of security targets. For example, anticipated threats are described in the chapter "Security Environment." When retrieving threats, the function firstly searches for a tag which has the text "Security Environment" by utilizing the metadata. After finding the tag, it pursues the following tags and records the threat names and contents, cf., Fig. 2. 
Similarly, the relationships are mentioned in the chapter "Rationale" by tables or texts. Security targets give each component an abbreviation, e.g., T.xxx, O.xxx. The prefix 'T.' denotes threats and the prefix 'O.' denotes security objectives. Thus, when retrieving relationships, the function records abbreviations of threats, security objectives, security requirements, and security functions beforehand. After the preparatory pursuit, the function reads relationships in the tables or texts leaning on the marks, i.e., each abbreviation name (Fig. 2).

Thus, the improved ISEDS can provide the functions equivalent to former all functions by XMS and the interfaces. Moreover, response performance of the retrieval was remarkably improved by the DPP technology of XMS.

\section{Concluding Remarks}

In order to apply effectively database technologies to information security engineering, we have redesigned and redeveloped the security requirement management database with a schemaless native XML database. Since users of the improved database do not need to define a schema, the former problem of the security requirement management database was solved and its performance was also improved. The users can collect, manage, and utilize security specifications with any layout, format, and structure. The specifications which are described with the database can be considered to be the security specification certified by ISO/IEC 15408. This fact is verifiable by our formal verification technique [3.5].

\section{References}

1. Horie, D., Morimoto, S., and Cheng, J.: A Web User Interface of the Security Requirement Management Database Based on ISO/IEC 15408, Proceedings of the International Conference on Computational Science (ICCS'06), Lecture Notes in Computer Science, Vol. 3994, Springer-Verlag (2006) 797-804

2. ISO/IEC 15408 standard: Information Technology - Security Techniques - Evaluation Criteria for IT Security (1999)

3. Morimoto, S. and Cheng, J.: Patterning Protection Profiles by UML for Security Specifications, Proceedings of the International Conference on Computational Intelligence for Modelling, Control and Automation and International Conference on Intelligent Agents, Web Technologies and Internet Commerce Vol-2 (CIMCAIAWTIC'05), IEEE-CS (2005) 946-951

4. Morimoto, S., Horie, D., and Cheng, J.: A Security Requirement Management Database Based on ISO/IEC 15408, Proceedings of the International Conference on Computational Science and its Applications (ICCSA'06), Lecture Notes in Computer Science, Vol. 3982, Springer-Verlag (2006) 1-15

5. Morimoto, S., Shigematsu, S., Goto, Y., and Cheng, J.: Formal Verification of Security Specifications with Common Criteria, Proceedings of the 22nd Annual ACM Symposium on Applied Computing (SAC'07), ACM (2007) 1506-1512

6. World Wide Web Consortium: XQuery 1.0 and XPath 2.0 Formal Semantics, http://www.w3.org/TR/xquery-semantics/

7. Xpriori LLC: NeoCore XMS, http://www.xpriori.com/ 